\title{
Clinical Briefing: Functional Neurological Symptom Disorder
}

\section{Sarah M Ratcliffe}

\section{Abstract}

Functional Neurological Disorder (FNSD) (also known as Somatoform or Conversion Disorder) is a relatively common neurological condition. The symptoms vary, and can include both motor, sensory neurological signs. Whilst there are still misunderstandings, recent progress has been made as to the causes of FNSD within the area of functional imaging, helping to unravel the underlying aetiology and neurobiology of FNSD. Once diagnosed with FNSD, there is a range of management and treatment options available for people including physical therapy, pharmacotherapy and psychological therapies. There is a dearth of information within the nursing literature to educate and support current nursing practice and decision making about people with FNSD. Consequently, approaches to the nursing care and management are often ad hoc and rely on research from other health disciplines. Nonetheless, there is now an emerging body of evidence to support specific management pathways to treat and manage FNSD. Neuroscience nurses are in a key position to embrace such pathways and influence the care offered to people with FNSD within the inpatient neurology setting.

\section{Functional Neurological Symptom Disorder}

Functional Neurological Symptom Disorder (FNSD) is a set of neurological symptoms that are unexplained by neurological disease (Aybek et al 2008). One of the biggest misunderstandings about FNSD is that the symptoms are under the person's control and that there needs to be a psychological cause for the 
symptoms. Motor symptoms can include tremor, weakness or dystonia and may be referred to as Functional Motor Disorder (FMD). Whereas seizure type symptoms, such as impaired or loss of consciousness, may be described as Psychogenic NonEpileptic Seizures (PNES) and are often described as Dissociative Seizure (Goldstein et al 2015). Other symptoms can include sensory loss and speech disturbances (Kremm 2004). FNSD is also associated with poor quality of life (Mitchell et al 2012), poor socioeconomic outlook (Carson et al 2011) and long term disability (Saifee et al 2012). Given the assumed psychological nature of FNSD, patients rarely present to a psychiatrist but often present within the neurological setting; frequently rejecting any psychological explanation for the causes of their symptoms (Stone et al 2010). Suzanne O'Sullivan (2016) described her experience of caring for FND patients in a series of case studies and found that many patients became angry when discussing the possibility of psychological causes to symptoms. Nevertheless,

attribution of symptoms to stress or emotional state has been associated with improved patient outcomes such as reduction in physical symptoms (Saifee et al 2012).

\section{Diagnosis of FNSD}

Alternative titles for FNSD have been adopted over the years, such as 'medically unexplained symptoms', 'functional symptoms', and 'non organic' symptoms. Over the last couple of decades, the term 'Conversion Disorder' has commonly been used. These terms have been used interchangeably and agreement on the correct term has remained undecided. However, many historical terms are now accepted as outdated. For example, until 1968 conversion symptoms were described as 
hysteria; now often considered pejorative in nature (Feinstein 2011).

The International Classification of Disease (ICD -10) (WHO 1992) and the Diagnostic Statistical Manual (DSM-5) (APA 2013) are both used by health professionals to diagnose illness. Both these diagnostic tools have noted the psychological causation of medically unexplained neurological symptoms. Up until recently the previous DSM-IV (APA 2000) stated that for a diagnosis of Conversion Disorder (CD) there had to be a notable psychological stressor. Similarly, the ICD-10 (WHO 1992) classified symptoms, such as unexplained weakness or seizures, in the Mental and Behavioural disorders section under Dissociative (Conversion) Disorders. However, following prolonged debate changes have been made to the diagnostic criteria and naming of $C D$ resulting in the preferred term of Functional Neurological Symptom Disorder (FNSD) being adopted in the DSM-5 (APA 2013) and psychological distress is no longer a diagnostic requirement as in the previous DSM-IV (see table 1). The World Health Organisation is due to publish the revised ICD version 11 in 2017 and there is a growing interest in re-classifying FNSD so it is included with other neurological disorders as well as psychiatric disorders. A key rational for this change is that functional disorders are one of the most common diagnoses in neurologic practice (Stone et al 2014,) and the acceptance that many patients presenting with unexplained neurological symptoms often deny any psychological distress. This diagnostic revision also emphasises the importance of a thorough neurological and psychological examination to identify positive signs of FNSD, rather than a lack of diagnosis through numerous clinical investigations. Possibly an influencing factor on why the medical community are unable to agree a standard label is the different theories and beliefs as to the causes of FNSD. Notwithstanding the recent 
diagnostic revision, defining and understanding FNSD continues to be problematic as many terms are still used interchangeably within the medical literature.

Table 1

\section{DSM 5 (APA 2013): Diagnostic criteria for}

\section{Functional Neurological Symptom Disorder (Conversion Disorder)}

A One or more symptoms of altered voluntary or sensory function

B Clinical findings provide evidence of incompatibility between the symptom and recognised neurological or mental condition

C The symptom or deficit is not better explained by another medical or mental disorder

D The symptom or deficit causes clinically significant distress or impairment in social, occupational, or other important areas of functioning or warrants medical evaluation.

Psychological factors: often present but not necessary

Nonetheless, significant progress has been made in supporting the diagnosis of FNSD. In criteria B above, the emphasis is on finding positive evidence to support the diagnosis of FNSD and to identify key clinical findings which may demonstrate evidence of incompatibility with neurological disease. For example, Hoover's sign, which is when weakness of hip extension returns to normal strength with contralateral hip flexion against resistance. Similarly, a unilateral tremor may be identified as functional if the tremor changes when the person is distracted (APA 2013). Roper et al (2013) advocate the tremor entrainment test as particularly significant in diagnosing functional tremor. 
Investigations, including blood tests, Magnetic Resonance Imaging (MRI) and Computerised Tomography (CT) imaging, as well as more invasive exploration such as electromyography are often undertaken to exclude other co-morbidities as well as to inform the diagnosis of FNSD.

\section{Aetiology}

A useful biopsychosocial explanation for FNSD is offered by Silver (1996) who suggests that the cause can often be understood as.... 'the result of an interaction among intra-psychic conflicts, cultural beliefs, beliefs about illness and learned maladaptive behaviour'(page134). FNSD is considered a psychiatric condition and one of the dominant explanations offered to many patients diagnosed with FNSD is that the physical symptoms are often caused by a psychological stressor or traumatic life event. Many patients dislike psychological causation attributed to their symptoms and many people are offended by the stigma of a psychiatric diagnosis (Kent and McMillan 2009). Possibly reinforcing this stigma is the belief by some that the person with FNSD is malingering due to the absence of pathology (Kanaan 2011). The DSM-5 describes malingering as... 'the intentional production of false or grossly exaggerated physical or psychological problems' (page 326). The motivation for malingering can include reasons such as obtaining financial compensation or evading criminal prosecution. Whilst 'malingering' is acknowledged in a minority of patients in the main this is an outdated view. 
Nevertheless, given that FNSD is rarely given the recognition of other neurological illness, this may reinforce the negative response that many patients often report feeling (Nicholson 2005).

Whilst the aetiology of FNSD has previously been poorly understood, there are numerous theories explaining FNSD with some converging empirical evidence to support them. Interestingly people with FNSD have a higher frequency of comorbid depressive and anxiety symptoms compared to the general population (Sar et al 2004). There is also an association with functional symptoms and negative life events, mental illness, and sociocultural factors, such as low socio-economic status (Nicholson 2005). Negative life events could include could be historical life events, such as childhood sexual abuse (Roelofs et al 2002) or recent stressful or traumatic events, ranging from such things as academic pressures to acts of terrorism (Hassett and Sigal 2002). Interestingly, Guerriero et al (2014) noted a 3-fold increase in children suffering from functional neurological symptoms following the Boston Marathon bombings. Parees et al (2014) found that $80 \%$ of 50 patients interviewed had experienced a physical event temporally related to the onset of Functional Motor Disorder (FMD) symptoms. The most common event was physical, but other events included drug reactions, infections, and exacerbation of a chronic pain. Whilst the above explore precipitating factors prior to onset of abnormal motor function Stone et al (2012) identified that the majority of patient's symptoms appeared suddenly rather than a gradual onset. Nevertheless, there is a significant group of patients for whom a psychological stressor has not been identified (Stone et al 2010). 
Finally, there is some emerging research using functional MRI (fMRI) scanning which considers a neurobiological basis for FNSD, especially in patients displaying abnormal motor function. Many of the studies focus on regions of the brain that control and process attention, volition and the bodies intention to move. Perez et al (2015) suggest alterations in neurocircuits which control emotional processing and awareness, and perception, such as the anterior cingulate and amygdala, and motor preparation and co-ordination, such as the supplementary motor area and cerebellum. Spence et al (2000) identified as significant the role of the dorsolateral prefrontal cortex in volition and that in two patients with functional paralysis this area of the brain was hypoactive. Whilst the sample size is small later studies have identified specific areas of the brain that play a potential role in intention to move could be inhibited in people with FNSD. Maurer et al (2016) explored the idea that people diagnosed with FMD report their abnormal motor symptoms as involuntary. Focusing on regions of the brain that control movement and the will to move, they found reduced connectivity between the right temporo-parietal junction (rTPJ) and the sensori-motor areas of the brain in 35 patients with FMD compared to an equivalent number of healthy controls. Baek et al (2017) consider an impairment in explicit intentional processes in people with FNSD. Their research identified that awareness was impaired between patients' voluntary intention to move and the awareness of the movement itself. In particular, they too identified reduced activity within the rTPJ in particular the inferior parietal lobule.

Our understanding of psychogenic non-epileptic seizures (PNES) has also developed recently. Kanaan et al (2007) cautiously identified a connection between 
traumatic life events and neurological symptoms, such as psychogenic seizures. There is now converging evidence that PNES is best considered a dissociative/arousal response similar to panic disorder. Hendrickson et al (2014) identified a higher proportion of patients with PNES suffer panic like symptoms compared to people with a diagnosis of epilepsy. Reuber and Brown (2016) consider a biopsychosocial approach to understanding PNES using an Integrative Cognitive model to explain aetiology. In their review of evidence they identified PNES patients had a greater recall of ictal panic symptoms and that the seizures may provide some relief from heightened arousal.

\section{Epidemiology}

Functional neurological symptoms are one of the most common diagnosis made in neurology (Stone et al 2014) although the information regarding the prevalence of FNSD differs greatly within the medical literature. Within the acute neurological setting the number of people presenting with FNSD symptoms increases significantly (Carson et al 2012). Some report suggest that CD is as common as other neurological conditions such as Parkinson's disease and Multiple Sclerosis (Stone et al 2009, Carson et al 2012). The Scottish Neurological Symptoms Study (2005) found that approximately one third of 3781 patients seen in a neurology outpatient's clinic had medically unexplained symptoms. There also appears to be some gender differences in the literature. For example, up to a third of neurology referrals are predominantly women (Carson et al 2000). Stone et al (2010) found that those with medically unexplained neurological symptoms were more likely to be younger females, many with greater mental disability and distress, and 
displayed a large number of symptoms. Nonetheless, there is also a significant number of people that do not display or report any psychological stressors at all.

\section{Management of FNSD}

Up until recently there has not been a strong evidence base to guide the treatment and management of patients with FNSD. Without clear guidance to care there is a real possibility that patient outcomes remain poor, the symptoms patients experience can become chronic (Carson 2003) and nurses may feel increasingly frustrated when caring for people with FNSD. Management of FNSD often depends on several factors, such as type and severity of symptoms and length of time since diagnosis. There is some recognition that antidepressant therapy and analgesia can help people with FNSD cope with the symptoms of this debilitating illness (Hatcher and Arroll 2008). However, pharmacotherapy alone will not necessarily improve symptoms.

Physiotherapy and occupational therapy are key interventions in the treatment for people who have FNSD with Functional Motor Symptoms (FMS) (Edwards 2012). Delargy et al (1986), whilst an old study, identified 6 case studies whereby 8-week neurological in-patient rehabilitation programme had been successful in people with medically unexplained paraplegia and quadriplegia. Czarnecki et al (2011) found that an intensive week long out-patient rehabilitation programme involving physiotherapy and occupational improved long-term outcomes for patients with Functional Motor Disorder (FMD). The rehabilitation involved a behavioural 
approach of motor re-programming rather than general physiotherapy thereby helping patients to reconstruct abnormal motor patterns. Long-term treatment outcomes identified that over $60 \%$ patients had improved or had maintained normal movement patterns. A similar study undertaken by Saifee et al (2012) which involved a combined cognitive and physical rehabilitation programme over a 4week period recognised the success of inpatient rehabilitation on patients diagnosed FMS. Saifee et al (2012) assert that attribution of symptoms to stress or emotional state has been associated with improved patient outcomes and as such patients not accepting the rational for cognitive behavioural therapy (CBT) as part of the rehabilitation programme were not accepted. Whereby the CBT focused on developing coping strategies and changing illness beliefs rather than reattributing symptoms to previous psychological stress or trauma. Out of 26 patients studied, $58 \%$ reported a benefit from the rehabilitation programme. A randomised controlled trial (RCT) was undertaken by Jordbru et al (2014) to explore the usefulness of physical rehabilitation in psychogenic gait. The authors found that a 3-week rehabilitation programme was effective in improving psychogenic gait relative to an untreated group, including at 1 year follow up. What was particularly interesting about this study was the use of the multidisciplinary team within the intervention. Many of the techniques described by Jordbru et al (2014) could easily be adopted by neuroscience nurses within the inpatient setting. For example, encouraging and reinforcing normal function was an integral part of the intervention, as well as minimising the attention given to sickness or illness behaviours. An interesting feasibility study undertaken by Nielsen et al (2016) found that an organised targeted programme of physical rehabilitation taken over a 5-day period improved outcomes for patients with Functional Motor Symptoms 
compared to a non-standardised treatment programme. Just over three quarters of the patients rated their symptoms as improved compared to just $18 \%$ of the control group. The authors are also quick to note that the improvement was observed in patients with characteristics usually associated with poor prognosis.

Whilst the evidence base for psychological therapies is limited for patients with FNSD, interventions such as CBT have been gaining ground over recent years. Conwill et al (2013) found some improvement in patients with functional neurological symptoms following group CBT sessions. Goldstein et al (2010) investigated the effectiveness of CBT on patients who suffer psychogenic nonepileptic seizures (PNES). Thirty-three patients underwent 3 months of CBT, plus Standard Medical Care (SMC), compared to 31 who received SMC alone. The results of the pilot study indicate that CBT is effective in reducing the number of seizures in patients who suffer from PNES. Following the success of this pilot study a longitudinal study has commenced, funded by the National Institute for Health Research (NIHR), due for completion in 2019, exploring the effectiveness of CBT as opposed to conventional medical intervention with people who suffer from dissociative seizures (Goldstein et al 2015).

More recently, there has also been a shift been towards self-help books which adopts the central principles of CBT, for use in patients with FNSD (Sharpe at al 2011). Whilst few studies have conclusively identified the benefit of psychosocial interventions for the management of FNSD, for CBT and other psychosocial interventions to be successful the patient must have a willingness to consider their symptom management in a psychological context as opposed to a purely physical 
one.

Effective and clear communication is key following a confirmation of a diagnosis of FNSD (Stone 2014). In her case studies O'Sullivan (2016) often reflects on the precise words and phrases she used to engage in discussion with patients. Many studies identify the importance of developing a therapeutic relationship with the patient as part of their management plan. For example, Maynard (2003) maintains that an initial negative response towards the patient can be detrimental and potentially cause further harm to the patient. Whilst Stone et al (2005) suggests that being non offensive when describing the diagnosis may even be enough to produce improvement of symptoms. Stone (2014) expressed the importance of the neurological consultation itself to be therapeutic for patients with FND and advocates neurologists use the principles of $\mathrm{CBT}$ in the consultation itself and that this alone can improve symptoms in some patients. For example, showing patients with functional motor symptoms their physical signs, such as Hoover's sign, can be useful in helping the patient come to terms with their diagnosis and the potential for reversing their symptoms, albeit if done in a sensitive manner (Stone and Edwards 2011).

Much work has been undertaken in Scotland over the past two decades appears to be leading the way in recognising the seriousness of this debilitating condition. The Stepped Care for Functional Neurological Symptoms report, published by Health Improvement Scotland (2012), was instrumental in helping improve the care for people with FND in Scotland. The guidance acknowledges the healthcare costs of FNSD to be approximately $£ 11.3$ million, excluding disability payments and social care costs. By drawing upon research from other professions allied to health, such as physiotherapy, occupational therapy and psychology, it is evident a 
multidisciplinary approach to care is advocated. Indeed, the key aims of the guidance is to educate health care workers, guide treatment and management, and thereby improving overall care for people suffering from FNSD. Furthermore, Nielsen et al (2015) have produced a set of recommendations for Functional Motor Symptoms that may go some way in also helping neuroscience nurses when caring for patients with FNSD. Whilst the guidance is aimed primarily at physiotherapists the recommendations can easily be extrapolated to nursing care as well as promoting inter-professional working.

Effective communication skills are inherent in nursing care and nurses could very easily adopt the strategies advocated by Stone $(2005,2014)$ and Nielsen et al (2015) when caring for patients diagnosed with FNSD. Nurses are seen as trustworthy, an essential attribute in caring for patients who distrust others and suffer with the symptoms associated with abuse or trauma. In the absence of a clear management plan a collaborative approach to care is recommended, including a specialist and tragetd rehabilitation service involving a cognitive behavioural approach. Overall, what is vital, is an acknowledgement of FNSD as a recognised neurological disorder.

\section{Attitudes of healthcare professionals towards people with FNSD}

Attitudes towards patents with FNSD are changing in the UK in part due to the work undertaken by a small group of psychiatrists and neurologist based in Scotland, specifically over the last two decades. Whilst this change remains incredibly slow there is now growing information available for patients with FNSD and health care professionals working with patients who have FNSD. For example, there has an increase in the use of self-help resources as well as some useful websites such as 
www.neurosymptoms.org and www.fndhope.org/ aimed at both professionals and patients.

Personal and professional beliefs can often determine how we perceive a person's situation. This professional judgement is paramount in the diagnostic process. O'Sullivan (2016) suggests that physicians often assert the seriousness of an illness not necessarily by how distressing the patient finds it, but by their own idea of what constitutes a serious illness. Kannan et al (2011) surveyed 349 neurologists in the UK and found that older neurologists were more inclined to infer an element of malingering in relation to functional neurological symptoms whilst younger, female neurologists tended to discuss psychological views (supporting the psychological model) and were more inclined to discuss this with their patients. Further, Kanaan (2011) also found that $63 \%$ of neurologists surveyed expected never to understand FNSD to be neurological in the same way as other neurological disorders such as Multiple Sclerosis (MS). Interestingly just over half of the 62 female neurologists surveyed did think it was neurological in the same way MS was or at least expected to think that one day.

Anecdotally the attitudes of nursing staff towards patients diagnosed with FNSD has been negative, but disappointingly very little research has been undertaken within this area. A small study undertaken by Ahern et al (2009) found that negative attitudes towards patients with FNSD from qualified and unqualified nursing staff were not uncommon. More specifically, a significant number of nurses found FNSD patients to be manipulative (46\%), annoying (24\%) and that neurology was an inappropriate place to nurse this group of patients (34\%). Nevertheless, 1 in 6 of 
the respondents thought that the symptoms displayed by patients were not real, and 1 in 10 that the patients were wasting time and did not deserve the same level of care as those patients who had other neurological illness. Interestingly, selfreported levels of knowledge of FND symptoms were low and negative attitudes of FND correlated with lower grade staff. Yon et al (2015) found that patients with medically unexplained symptoms generated feelings of frustration, anxiety and self-perceived lack of competency in a small number of junior doctors in the UK. Remarkably these junior doctors also reported negative attitudes of some senior clinicians, which is concerning given the potential for senior clinicians to act as role models for their junior colleagues.

\section{Conclusion}

FNSD remains one of the most commonly diagnosed neurological conditions. Whilst developments have been made over the last two decades' progress is slow within the field of nursing, and there remains a disappointing amount of literature to guide nursing practice and decision making. There remains a stigma and negative attitudes towards patients who have this debilitating condition, which is not helped by historical notions of hysteria and the use of previous pejorative terms to explain its cause. There continues to be professional ambiguity about whether patients with FNSD are legitimate neurological patients and whether the neurological setting is an appropriate area of care for people with FNSD. 
Nevertheless, exciting progress has been made which supports aetiological theories as to the causes of FNSD, especially within the field of functional imaging. More evidence is needed to support the management of patients with FNSD but it is generally agreed that a multidisciplinary approach is required which includes both physical and psychological therapies. It's imperative that neurologists offer a clearer explanation to patients and offer greater support to MDT colleagues for rehabilitation to be more successful. Given that $63 \%$ of neurologists do not necessarily acknowledge FNSD as neurological this may pose a significant challenge.

\section{Future challenges and recommendations}

Considerable steps need to be made for FNSD to be recognised as a genuine neurological condition and assumed the same importance as other neurological conditions such as Multiple Sclerosis and Parkinson's disease. Given the poor level of knowledge in some nursing staff and ambiguity with other health professionals this may prove challenging.

There is an urgent need to improve education to equip neuroscience nurses with the necessary knowledge and skills to effectively and confidently manage patients with FNSD and to avoid negative attitudes being spread. Given that neuroscience nurses have much more contact with patients than other professionals they are in a good position to reinforce management plans and knowledge of FNSD, such as that recommended by Nielsen et al (2015). Moreover, approaching the care of people with FNSD with the same mind-set afforded to people with other neurological illness and adopting a goal setting approach to management may go 
some way in improving the patients' experience.

Neuroscience nurses should pay close attention to Nielsen et al (2015) recommendations and positively reinforce normal function whilst acknowledging and legitimising symptoms whilst avoiding positively reinforcing dysfunction. This can be done when engaging with patients during daily activities of living. To assist with communication nurses could learn and use the principles of CBT when communicating on a daily basis with FNSD patients. Due to their close contact with inpatients nurse are able to recognise and challenge unhelpful thoughts and behaviours. Moreover, the correct use of terminology is so important when conversing with patients, avoiding the use of terms that could lead to feelings of suspicion.

Neuroscience nurses should embrace research opportunities within interprofessional teams as well as consider undertaking their own exploration of this under-researched area. Given that FNSD is one of the most common neurological diagnosis it would be pertinent to include this subject within post-qualifying neuroscience nursing curricula.

Improvements in care can be made with swift access to neurological care, a combination of physical and cognitive rehabilitation and pharmacotherapy, and by offering empathy and genuineness by all staff that encounter people with FNSD. Research literature regarding care and management of patients with FNSD within the nursing press is minimal but nurses can extrapolate findings from medical neuroscience resources and other allied healthcare professionals to help aid the 
nursing care and management of FNSD. What is acutely transparent is the importance of inter-professional working to improve the care FNSD patients receive. This may go some way in removing the apparent negative attitude towards this group of patients. 


\section{References}

Ahern, L., Stone, J. and Sharpe, M.C. (2009) Attitudes of neuroscience nurses towards patients with conversion symptoms Psychosomatics. 50, 336-339.

American Psychiatric Association (2000) Diagnostic and statistical manual of mental disorders (4th edition) (text revision) American Psychiatric Association Washington.

American Psychiatric Association (2013) Diagnostic and statistical manual of mental disorders (5th edition) (text revision) American Psychiatric Association Washington.

Aybek, S., Kanaan, R. and David, A.S. (2008) The neuropsychiatry of conversion disorder. Current Opinon in Psychiatry 21,275-280.

Baek, K., Doñamayor, N., Morris, L.S., Strelchuk, D., Mitchell, S., Mikheenko, Y., Yeoh, S.Y., Phillips, W., Zandi, M., Jenaway, A., Walsh, C. and Voon, V. (2017) Impaired awareness of motor intention in functional neurological disorder: implications for voluntary and functional movement. Psychological Medicine 2017; :1-13.

Carson, A.J., Brown, R., David, A.S., Duncan, R., Edwards, A.J., Goldstein, L.H., Grunewald, R., Howlett, S., Kanaan, R., Mellers, J., Nicholson, T.R., Reuber, M., Schrag, A.E., Stone, J. and Voon, V. (2012) Functional (conversion) neurological symptoms: reseach since the millennium Journal of Neurology, Neurosurgery and Psychiatry. 83, 842-850.

Carson, et al (2011) Disability, distress and unemployment in neurology outpatients with symptoms unexplained by organic disease. JNNP 82, 810-81

Carson, A.J., Best, S., Postma, J. et al (2003) The outcome of neurology outpatients with medically unexplained symptoms: a prosepctive cohort study. Journal of 
Neurology, Neurosurgery and Psychiatry. 74, 897-900.

Carson, A.J., Ringbauer, B., Stone, J., McKenzie, L., Warlow, C. and Sharpe, M. (2000) Do medically unexplained symptoms matter? A prospective cohort study of 300 new referrals to neurology outpatients clinics. Journal of Neurology, Neurosurgery and Psychiatry 68, 207-210

Conwill, M., Oakley, L., Evan, K. and Cavanna, A.E. (2014) CBT-based group therapy intervention for non-epileptic attacks and other functional neuological symptoms: a pilot study. Epilepsy and Behaviour. 34, 68-72.

Czarnecki, K., Thompson, J.M., Seime, R., Geda, Y.E., Duffy, J.R. and Ahlskog, J.E. (2011) Functional movement disorders: successful treatment with a physical therapy rehabilitation protocol. Parkisnonism and Related Disorders. 18, 247-251. Delargy, M.A., Peatfield, R.C. and Burt, A.A. (1986) Successful rehabilitation in conversion paralysis. British Medical Journal. 292, 1730-1731.

Feinstein, A. (2011) Conversion disorder: advances in our undertsanding. Canadian Medical Association Journal. 183,8,915-919.

Goldstein, L.H., Mellers, J.D.C., Landau, S., Stone, J., Carson, A., Medford, N., Reuber, M., Richardson, M., McCrone, P., Murray, J. and Cha;der, T. (2015) COgnitive behavioural therapy vs standardised medical care for adults with dissociative non-epileptic seizures (CODES): a multicentre randomised controlled trial protocol. BMC Neurology 15:98,

Goldstein, L.H., Chalder, T., Chigwedere, C., Khondoker, M.R., Moriarty, J., Toone, B.K., and Mellers, J.D.C. (2010) Cognitive-behavioral therapy for psychogenic nonepileptic seizures: a pilot RCT. Neurology 74,1986-94.

Guerriero, R.M., Pier, D.B., de Gusmao, C.M., Bernson-Leung, M.E., Maski, K.P., Urion, D.K. and Waugh, J.L. (2014) Increased pediatric functional neurological 
symptom disorders after the marathon bombings: a case series. Pediatric Neurology 51, 619-623.

Hassett, A.L. and Sigal, L.H. (2002) Unforseen consequences of terrorism: medically unexplained symptoms in a time of fear. Archive of Internal Medicine 162, 18091813.

Hatcher, S. and Arroll, B. (2008) Assessment and management of medically unexplained symptoms. British Medical Journal 336,1124-1128.

Hendrickson, R., Popescu, A., Dixit, R., Ghearing, G. and Bagic, A. (2014) Panic attack symptoms differentiate patients with epilepsy from those with psychogenic nonepileptic spells (PNES). Epilepsy Behaviour 37,210-4.

Jordbru, A.A., Smedstad, L.M., Klungsøyr, O. and Martinsen, E.W. (2014) Psychogenic gait disorder: A randomized controlled trial of physical rehabilitation with one-year follow-up. Journal of Rehabilitaion Medicine. 46,181-7.

Kanaan, R.A., Armstrong, D. and Wessley, S.C. (2011) Neurologist's understanding and management of conversion disorder. Journal of Neurology, Neurosurgery and Psychiatry. 82, 961-966

Kanaan, R., Craig, T.J.K., Wessely, S.C. and David, A.S. (2007) Imaging repressed memories in motor conversion disorder Psychosomatic Medicine 69,202-205.

Kent, C. and McMillan, G. (2009) A CBT-based approach to medically unexplained symptoms. Advances in Psychiatric Treatment. 15,146-151.

Kremm (2004)

Maurer CW, LaFaver K, Ameli R, Epstein SA, Hallett M, Horovitz SG. (2016) Impaired self-agency in functional movement disorders: A resting-state fMRI study. Neurology 1-8.

Mitchell, J., Ali, F., and Cavanna, A.E., Dissociative experiences and quality of life in 
patients with non-epileptic attack disorder. Epilepsy Behaviour 25,307-12.

Nicholson, T.R.J, Stone, J. and Kanaan, R.A.A. (2011) Conversion Disorder: a problematic diagnosis. Journal of Neurology, Neurosurgery and Psychiatry 82,12671273.

Nicholson, S.M. (2005) An exploration of the predisposing factors of conversion disorder and why this disorder should not be classed as a mental illness (Unpublished) University of Salford.

Nielsen, G., Stone, S., Matthews, A., Brown, M., Sparkes, C., Farmer, R., Masterton, L., Duncan, L., Winters, A., Daniell, L., Lumsden, C., Carson, A., David, A.S. and Edwards, M. (2015) Physiotherapy for functional motor disorders: a consensus recommendation Journal of Neurology, Neurosurgery and Psychiatry. 86,11131119

Nielsen, G., Buszewicz, M., Stevenson, F., Hunter, R., Holt, K., Dudziec, M., Ricciardi, L., Marsden, J., Joyce, E. and Edwards, M.J. (2016) Randomised feasibility study of physiotherapy for patients with functional motor symptoms. Journal of Neurology, Neurosurgery and Psychiatry. 0,1-7.

O'Sullivan, S. (2016) It's all in your head. True stories of imaginary illness. London, Vintage.

Parees, I., Kojovic, M., Pires, M., Agusti, I.R., Saifee, T.A., Sadnicka, A., Kassavetis, P., Macerollo, A., Bhatia, K.P., Carson, A., Stone, J. and Edwards, M.J. (2012) Physical precipitating factors in functional movement disorders. Journal of the Neurological Sciences 338,174-177

Perez, D.L., Dworetzky, B.A., Dickerson, B.C., Leung, L., Cohn, R., Baslet, G. and Silbersweig, D.A. (2015) An integrative neurocircuit perspective on psychogenic nonepileptic seizures and functional movement disorder: neural functional 
unawareness. Clinical EEG and Neuroscience. 46,1,4-15.

Reuber, M. and Brown, R.J. (2016) Understanding psychogenic nonepileptic seizures-Phenomenology, semiology and the Integrative Cognitive Model. Seizure $44,199-205$

Roelefs, K., Keijers, G.P.J., Hoogduin, K.A.L., Naring, G.W.B, and Moene, F.C. (2002) Childhood abuse in patients with conversion disorder. American Journal of Psychiatry. 159, 1908-1913.

Saifee, T.A., Kassavetis, P., Parees, I., Kojovic, M., Fisher, L., Morton, L., Foong, J., Price, G., Joyce, E.M. and Edwards, M.J. (2012) Inpatient treatment of functional motor symptoms: a long term follow up study. Journal of Neurology. 259,19581963

Sar, V., Akyuz, G., Kundakci, T. Kiziltan, E. and Dogan, O. (2004) Childhood trauma, dissociation and psychiatric co-morbidity in patients with conversion disorder. American Journal of Psychiatry 161,2271-2276.

Silver, F. (1996) Management of conversion disorder American Journal of Physical Medicine and Rehabilitation 75, 134-140

Sharper, M., Walker, J., Williams, C., Stone, J., Cavanagh, J., Murray, G., Butcher, I., Duncan, R., Smith, S. and Carson, A. (2011) Guided self-help for functional (psychogenic) symptoms. Neurology. 77,6,564-572.

Spence, S.A., Crimlisk, H.L., Cope, H., Ron, M.A. and Grasby, P.M. (2000) Discrete neurophysiological correlates in prefrontal cortex during hysterical and feigned disorder of movement. Lancet, 355,1243-4.

Stone, J. (2014) Functional Neurological Disorder: The neurological assessment as treatment. Clinical Neurophysiology. 44, 363-373. 
Stone J, Edwards M. Trick or treat? Showing patients with functional (psychogenic) motor symptoms their physical signs. Neurology 2012; 79:282-4. Stone, J., Hallet, M., Carson, A., Bergen, D. and Raad, S. (2014) Functional disorders in the neurology section of the ICD-11 Neurology 83(24), 2299-2301.

Stone, J., Warlow, C. and Sharpe, M. (2012) Functional weakness: clues to mechanism from the nature of onset. Journal of Neurology, Neurosurgery and Psychiatry 83,67-9.

Stone, J., Warlow, C. and Sharpe, M. (2010) The symptom of functional weakness: a controlled study of 107 patients. Brain. 133, 1537-1551.

Stone, J., Carson, A., Duncan, R. et al (2009) Symptoms unexplained by organic disease in 1144 new neurology out-patients: how often does the diagnosis change at follow up? Brain, 132, 2878-88.

Stone, J. Carson, A. and Sharpe, M. (2005a) Functional Symptoms in Neurology: Management. Journal of Neurology, Neurosurgery and Psychiatry. 76 (Suppl I), 1321.

Yon K, Nettleton S, Walters K, et al Lamahewa, K. and Buszewicz, M. (2015) Junior doctors' experiences of managing patients with medically unexplained symptoms: a qualitative study British Medical Journal Open 5:e009593. doi: 10.1136/bmjopen2015-009593 